\title{
Analysis of Baidu Mobile Search Promotion in Wenzhou
}

\author{
Zhijie Xia \\ E-commerce, School of Economy and Trade, Zhejiang Industry \& Trade Vocational College, \\ Wenzhou, Zhejiang 325003
}

Keywords: Baidu Mobile; Baidu Mobile Promotion; Mobile Internet; Wenzhou

\begin{abstract}
With the onset of mobile internet, many companies and businesses engaged in PC-based website promotion are increasingly concerned about the mobile-based promotion of websites, along with the arrival of the mobile age, more attention should be given to mobile business opportunities. For businesses, it means both opportunity and challenge, Baidu search results are much trusted by users, so as to make the Baidu mobile search promotion recognized among the users. This thesis attempts to propose some workable solutions to address the deficiencies in the course of promotion and allow for the maximized business benefits with respect to the Baidu mobile promotion of key words and the analysis of present situation of Baidu mobile promotion in Wenzhou.
\end{abstract}

\section{Introduction}

Based on the customer service data provided by Zhejiang Guoji Internet Information Technology Co., Ltd., this thesis closely analyzes the Baidu mobile promotion in Wenzhou, identifies the deficiencies such as irrational configuration of keywords, incomplete display of the search results, establishes some workable solutions from the proposals through trial and error, and provides the case support for the subsequent mobile promotion.

\section{Present Situation of Baidu Mobile Promotion}

Baidu Mobile Promotion. Baidu, also known as the world's largest Chinese search engine and Chinese website, launched its Baidu Mobile Search Engine in $2011^{[1]}$. It is defined as the possible impression of the promotion information in the page of search results, when you search a keyword on Baidu Mobile Search via cellphone or tablet ${ }^{[2]}$. Based on the pay-per-click model and the configuration of keywords, a huge amount of search impressions will be delivered to businesses, which in turn stimulates the conversion of orders. The number of Baidu Mobile Search hit 400 million and the number of active users a month hit 100 million, in this age of internet, the ratio of Baidu mobile search promotion to mobile search has been 3:1, Baidu Mobile Search also adopts the paid listing model like its PC search, both are complementary, the marketing process comprises five steps: webpage display-click-visit-monitoring-optimization, impression:" 3 items on the top and 3 items on the bottom", ranking: the degree of quality * bid, charging: pay-per-click ${ }^{[3]}$.

Overview of Baidu Mobile Promotion. With the increase of internet users, many businesses focus on the construction of mobile websites. Fig. 1 shows the comparison of keyword searches for the recent hottest Descendants of the Sun, the mobile promotion is two thirds of the overall promotion, more and more users prefer searching different keywords on mobile devices, and the cost of mobile promotion is cheaper than that of PC. However, there is a limited availability of impressions, the mobile promotion will rank the webpages depending on individual searching habit and the quality of search keywords, based on the pay-per-click model, and the mobile promotion is only applicable to mobile devices, the display character and form of searched results will be more suitable to the cellphone screen, three impression locations are more highlighted. Furthermore, 
some are more concerned about the traffic flow, so the conversion ratio of mobile search is higher, the cost-per-click is reduced, and the consulting channels such as Baidu Shangqiao are available for use.

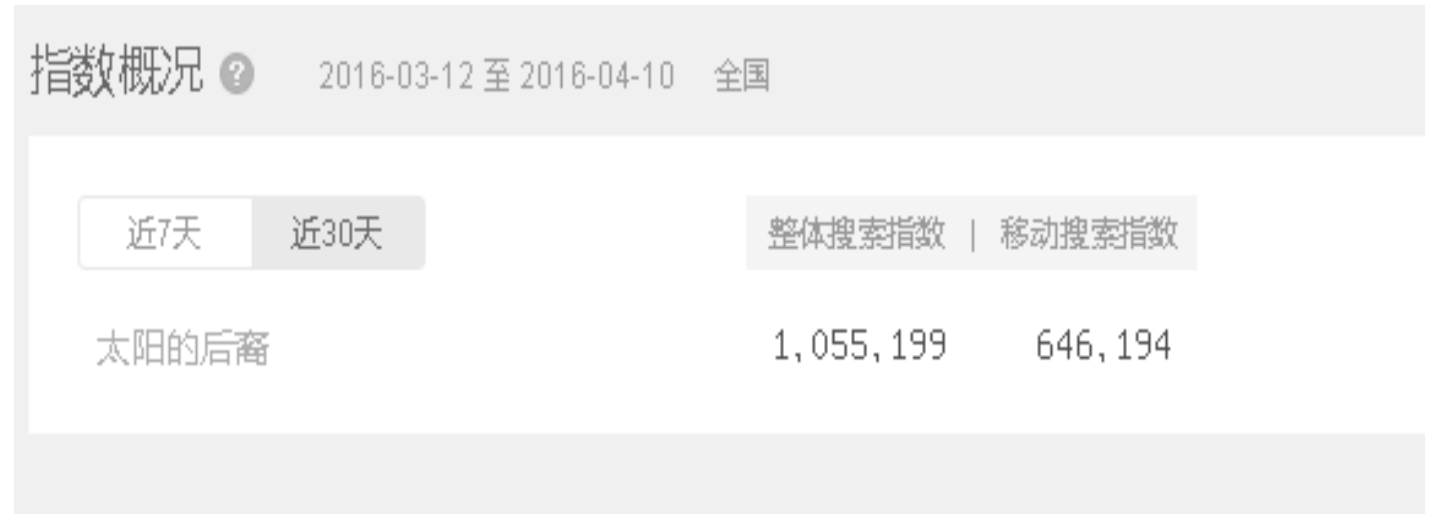

Figure 1. Comparison between the mobile search and overall search

\section{Problems in Baidu Mobile Promotion}

Invalid Mobile Search. Reason for the validity of Baidu mobile promotion: the delivery of a keyword in Baidu Mobile is lower than the minimum display price for its keyword promotion [4]. The invalidity of mobile search is caused by the low bid, two different prices and degrees of keyword quality will be designed for the same keyword, when the bid for a keyword is lower than RMB 1, it may generally lead to the invalid promotion, and the keyword will be only displayed on Baidu Mobile after an appropriate adjustment. Fig. 2 shows the relationship between the bid price for keyword and the display.

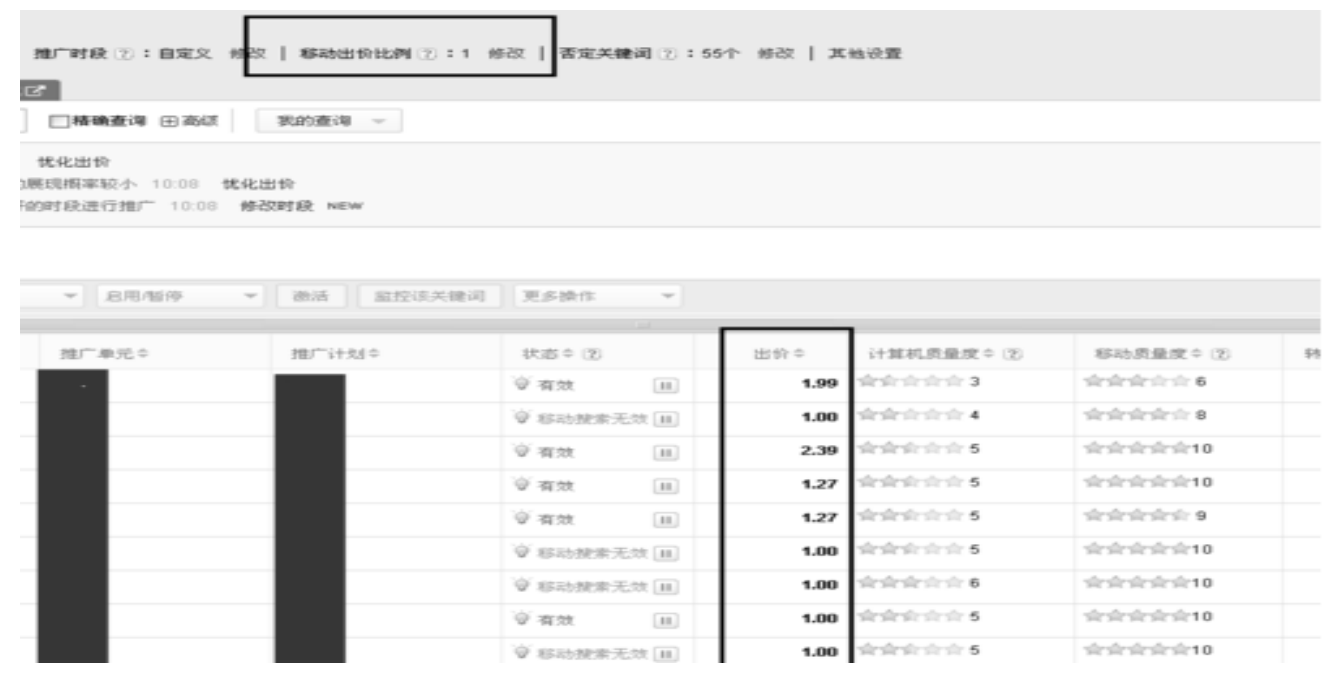

Figure 2. Relationship between the bid price for keyword and the display

Low Click Rate on the Impressions from Mobile Devices. With the popularity of smart phones, the mobile search volume is also increasing, the search volume from mobile devices is 3 times from PC, but the conversion ratio of searches from mobile devices is lower than that from PC. It can be attributed to different user scenarios, limitation of the mobile screen size, and generally, only three locations are available on the heads of a mobile device, while there are 10 on PC [5]. In addition, the clicks and impressions on webpages would substantially increase by considering the online user behavioral habits, promotion delivery location, promotion delivery time, and whether the titles, descriptions and pictures are attractive enough, assisted by direct calls, online consultation 
and Baidu Baiqiao. Firstly, APP has the highest priority, which may not appear simultaneously with other additional ideas. Secondly, phone calls and Baidu Shangqiao have the second highest priority, which may appear simultaneously or independently. Lastly, customers who participate in the E-point Pass callback experiment may seek the service of Baidu Shangqiao and E-point Pass by 50\%:50\% chance, without making a call.

Malicious Clicks from Mobile Devices. Everyone who engages in Baidu promotion has the same problem: for paid ads, malicious click is the primary headache, which is simply defined as: in order to suppress the competitors, some company repeatedly clicks on a webpage to diminish the advertising effect of a competitor, waste its money, make their ranking elevated and eliminate a competitor, which in turn reduces the competition cost. Fig.3 shows some signs of malicious click, e.g: the increase of daily clicks on a keyword, the increase of daily consumption, or excessive clicks from one IP.

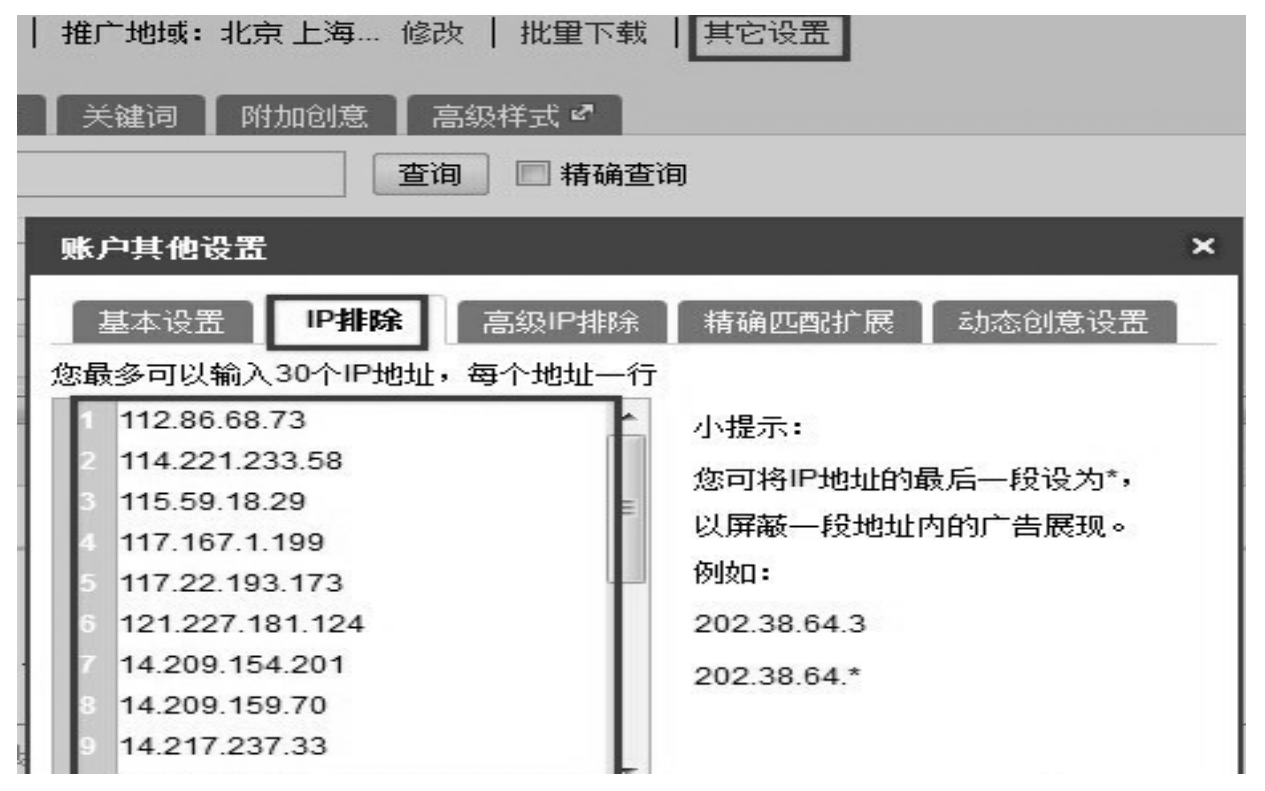

Figure 3. Blocking of the IPs as the source of malicious clicks

Underperformance of Mobile Promotion. Since mobile browsing behavior is intermittent, the browsing behavior tends to be fragmented. Firstly, user has no time to digest the advertising information or decide whether to buy or not; secondly, the screen size of a mobile device is limited, which means that product information may not be fully displayed and the advertising effect will be diminished to a large extent; thirdly, the user experience in mobile devices is poor in terms of real-time communication, which may lower the user interest for consultation and affect the promotion effect; lastly, many companies deliver large-size pictures on their sponsored ads, since the domestic data is expensive, user chooses to close the webpage to save the data cost, various factors have led to the underperformance of mobile promotion.

\section{Recommendations for Mobile Promotion}

Make more Valid Promotion by Optimizing the Keywords. Baidu Search has set strict requirements for the degree of keyword quality and the bid of keyword, when the bid of a keyword is lower than the average price for impressions, the information will not be displayed, also referred 
to as invalid promotion, which means that the bid of a keyword should be higher than the average price for impressions. There is a lot of marketable software used to modify the price of keyword software, for instance, adSage is designed specifically to Baidu backstage accounts, which allows for the automatic configuration of the display location of each keyword, impression price and bid area, or the manual modification of the bid for a keyword. After the configuration of keyword location and the effectiveness of a bid, it will reconfigure the setting of this keyword.

Improve the Information Impression by Regional Website Optimization. Mobile promotion is preferably made in local user searches, from Fig.4, when we search for a tourist attraction, Baidu Search displays lvmama.com on PC, while it mostly displays a list of Wenzhou local tourist attractions as shown in Fig.5 on mobile devices, the mobile users tend to search some questions with immediate solution: where is a parking lot nearby? So the location of your target customers is the key point, you can identify the areas with more potential customers during a period of advertising campaigns, or make a webpage optimization for local areas. Specifically, add the name of place in front of a keyword, or even modify the geographical location of your website [6].

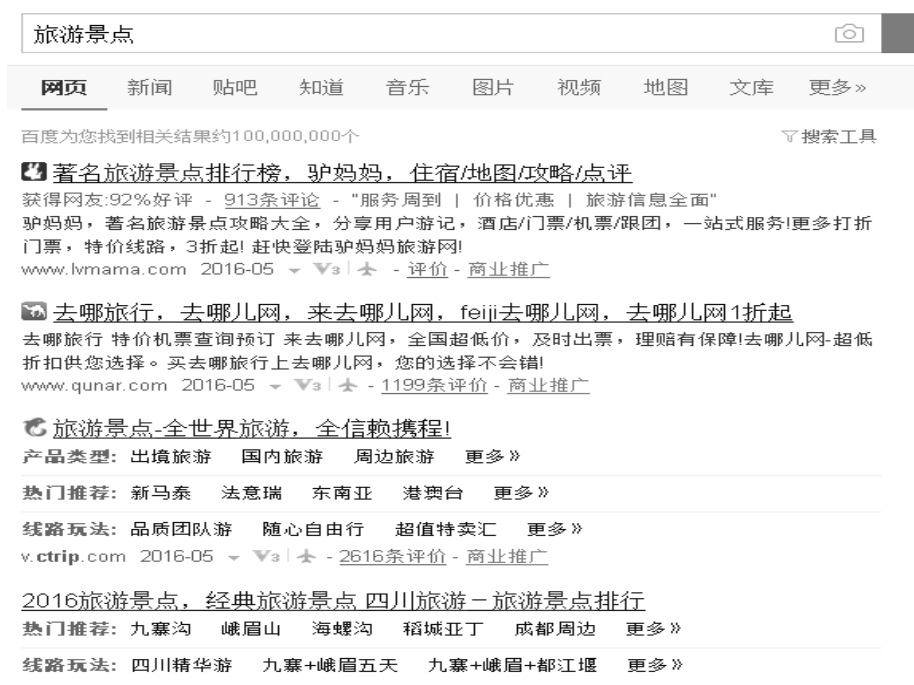

Figure 4. Impressions on PC

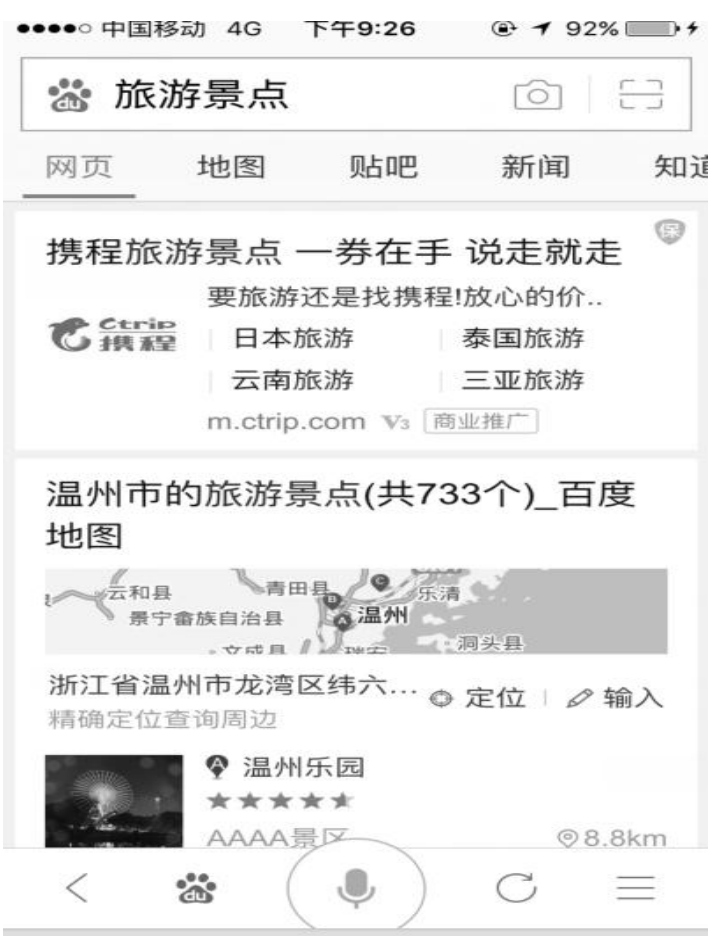

Figure 5. Impression on mobile devices 
Prevent Malicious Clicks by Backstage Settings. As to the malicious clicks in Baidu promotion, company may as well select the precise keywords, or set the planned budget by summing up the average daily consumption for each keyword, as shown in Fig.6 [7]. The promotion cost will be reduced by setting different budget for each keyword and lowering the rank. Fig.7 shows the Baidu backstage setup program which blocks the IPs considered as the source of malicious clicks.

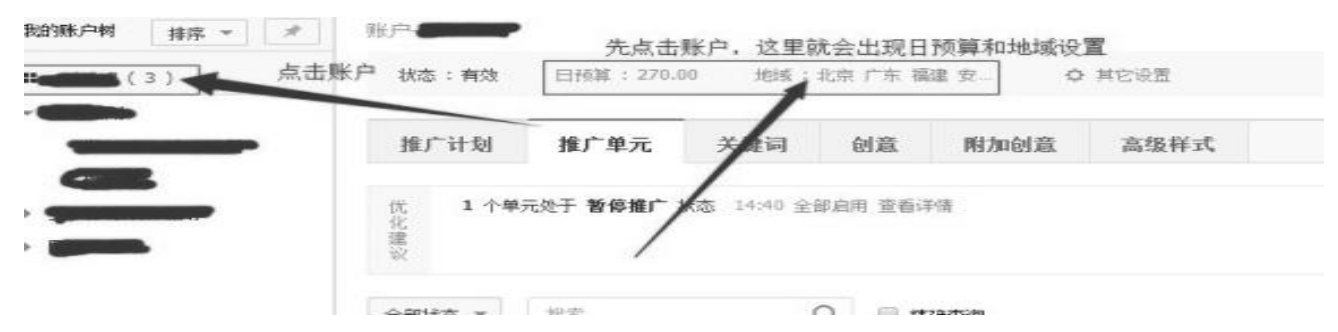

Figure 6. Setting of the daily budget and the intended promotion areas

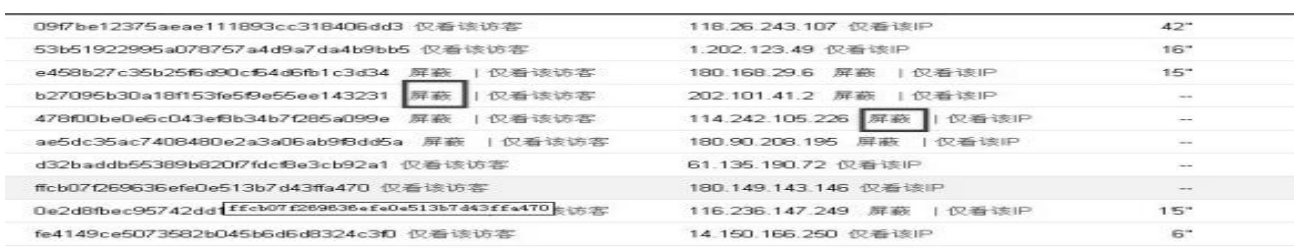

Figure 7. Blocking of the IPs considered as the source of malicious clicks

Understand the Customers, Optimize the Website, and Improve ROI. Prior to the mobile promotion, we need to understand our target customers, since it is mostly launched at noon, in evening, on weekends or holidays, the searching time frames are varied, the searches cover a wide range of areas, more customers already close the promotion information before they get to know the products or services of a company, user searching habits tend to be fragmented [8]. A promotion campaign should seize the dominant rank by price advantage, if the impression of a keyword attracts more clicks, company may as well raise the bid for this keyword; company should change the delivery time and areas depending on its target customers, through the analysis of their search behaviors. For the business mobile sites, the company profile on webpage should be clear and intelligible to target customers, so that user experience would be enhanced; the number of words on landing page should be more readable and creative, some GIFs may be added to be more appealing to customer clicks, Baidu Shangqiao and other business communication software should clearly state that customers are required to leave their QQ or mobile number for the convenience of follow-up contact, which is particularly important for the follow-up customer tracking on mobile devices [9].

\section{Summary}

It is not easy for mobile internet practitioners to perform well in paid mobile promotion, nor could this be realized overnight. The internet development indicates the importance of mobile maintenance of the mobile search promotion sector ${ }^{[10]}$. Here, some workable solutions such as optimization of keywords or backstage service are proposed to solve the specific problems that Zhejiang Guoji Internet Information Technology Co., Ltd.(Wenzhou) has encountered in mobile promotion, e.g.: low impression rate, invalid search, or malicious clicks. 
[1] Wu Yongyi.Mobile social marketing money scene:Business management[J]. 2013, (4): 115-116.

[2] Dai Guozhong,Chen Wei,Hong Wenxue,et al. Information visualization and visual analytics: challenges and opportunities [J]. Science China: Information Science, 2013,43(1):178 - 184.

[3] Chen D M,Tsai S S,Chandrasekhar V,et al. Tree histogram coding for mobile image matching[C]/ /Proceedings of the 2009 Data Compression Conference,March 16 - 18,2009: $143-152$.

[4] Jamie. The future of mobile search \& augmented reality [EB/OL]. [2013 - 08 - 26]. http://blog. pongr. Com/2010/01/image - recognition-augmented-reality.

[5] Marimon D,Adamek T,G llner K,et al. Mobile visual recognition, the future of mobile A R [EB/OL]. [2013 - 08 - 26]. http:/ /www. perey. com/MobileA R Summit/Telefonica R \& D-Mobile-Visual- R ecognition. pdf.

[6] Chen D,Tsai S,Chandrasekhar V,et al. R esidual enhanced visual vector as a compact signature for mobile visual search[J]. Signal Processing,2013(93):2316 -2327.

[7] Cao Y, Ritz C, Raad R. Image compression and retrieval for mobile visual search[C]/ /2012 International Symposium on Communications and Information Technologies(ISCIT), 2-5 Oct,2012,Gold Coast,OLD:1027-1032.

[8] Chen Wen feng,Xuan Yuming,Fu Xiaolan. Cognitive mechanism and application of visual search [J]. China Computer Society Newsletter, 2009,5(7):17 -22.

[9] Wang Changhu,Zhang Lei. Charm and challenge sketches search [J]. China Computer Society Newsletter, 2012, 8 (12):8-15.

[10]Lin Yun.Research on Baidu Inc mobile search profit model [J]. Beijing University of Posts and Telecommunications, 2014, 26-30. 